\title{
Preoperative Tumor Abnormal Protein as a Promising Biomarker to Predict Oncological Outcome of Hepatocellular Carcinoma After Curative Resection
}

\author{
Huayong Cai \\ Liaoning Cancer Institute and Hospital \\ Wenxin Li \\ Liaoning Cancer Institute and Hospital \\ Yu Zhang \\ Liaoning Cancer Institute and Hospital \\ Xiangdong Hua ( $\square$ hxd_Inzl@163.com ) \\ Liaoning Cancer Institute and Hospital
}

\section{Research Article}

Keywords: Tumor abnormal protein, HCC, nomogram, recurrence-free survival

Posted Date: September 8th, 2021

DOl: https://doi.org/10.21203/rs.3.rs-870375/v1

License: (c) (i) This work is licensed under a Creative Commons Attribution 4.0 International License.

Read Full License 


\section{Abstract}

Background: TAP (tumor abnormal protein) has been used as an important indicator in the early diagnosis of cancers, and some literatures showed that TAP can act as a prognostic factor in different kinds of cancer. The objective of this study was to explore the potential relationship between TAP and the prognosis of HCC after radical hepatectomy, and attempted to construct a robustly predictive nomogram on the strength of TAP and other prognostic variables of HCC patients.

Methods: This retrospective study included 168 HCC patients (tumor recurrence occurred in 78 patients) who had undergone curative resection during January 2018 to June 2020 at the Department of Hepatopancreatobiliary Surgery of Liaoning Cancer Hospital \& Institute. Serum TAP was detected by Abnormal Sugar Chain Structure of Glycoproteins, and according to the area of condensation particle, the whole population was categorized into the TAP high group $\left(\mathrm{TAP} \geq 225 \mu \mathrm{m}^{2}\right)$ and TAP low group $\left(\mathrm{TAP}<225 \mu \mathrm{m}^{2}\right)$.

Results: There was no correlation between maximum tumor size and TAP. In the whole population or subgroups stratified by maximum tumor size, the recurrence-free survival (RFS) rate of the TAP low group was distinctly higher than TAP high group $(\mathrm{P}<0.05$ for all). The multivariate analysis revealed that TAP (hazard ratio [HR], 3.47; 95\% Cl, 2.18-5.51; $\mathrm{P}<0.001)$, large tumor size (HR, 2.18; 95\% Cl, 1.36-3.49; $\mathrm{P}<0.001)$, poor tumor differentiation $(\mathrm{HR}, 0.53 ; 95 \% \mathrm{Cl}, 0.33-0.84 ; \mathrm{P}=0.007)$ and presence of microvascular invasion (MVI) (HR, 2.03; 95\% Cl, 1.28-3.22; $\mathrm{P}=0.003)$ were independently associated with RFS. The prognostic implication of nomogram incorporating TAP, maximun tumor diameter, tumor differentiation and MVI was stronger than the model that integrated maximun tumor diameter, degree of tumor differentiation and MVI only.

Conclusion: The present study suggested that higher preoperative TAP was correlated with undesirable prognosis in HCC patients who had undergone radical hepatectomy,

and on the strength of prognostic variables identified by multivariate analysis, we constructed a robust nomogram for RFS of postoperative HCC patients.

\section{Background}

Hepatocellular cancer (HCC) represents the most common primary liver malignancy, accounting for 75$82 \%$ of the cases histologically[1]. Although achieved some advances, curative resection is still the most optimal treatment for $\mathrm{HCC}[2]$. However, the high recurrence risk is a substantial challenge for HCC patients who had undergone radical hepatectomy, and the 5 years recurrence rate is higher than $50 \%[3]$. If we can identify high-risk HCC patients, and apply suitable treatments to protect them from tumor recurrence, the prognosis may be ameliorated.

Glycosylation is an important biochemical mechanism that can regulate cellular functions. In almost all kinds of cancer, abnormal glycosylation is common in many proteins, which has been shown to be 
correlated with tumor development, metastasis and the clinical outcome of patients[4-6]. Meezan et al[7] indicated for the first time that cancer-associated glycans were different from glycans of healthy cells. Besides, in normal tissues, lots of neoplasm-associated glycans are present at low levels, while tumors have higher levels[8]. Glycoproteins produced by various malignant tumors are collectively referred to tumor abnormal protein (TAP). Fortunately, with the progression of tumors, once TAP levels reach a given threshold, we can detect them in the peripheral blood. Currently, TAP is used as an important indicator in the early diagnosis of cancers. For example, Huang et al[9] demonstrated that TAP can be considered as a significant indicator for clinically monitoring colorectal cancer patients, and Ma et al[10]suggested that TAP has high accuracy in the diagnosis of early-stage endometrial cancer when combined with transvaginal ultrasound. In addition, a few literatures showed that TAP can act as a prognostic factor in different kinds of cancer, such as non-small cell lung cancer (NSCLC), gastric cancer and esophageal squamous cell carcinoma[11-13]. However, as far as we know, up to now, no data indicated that there was a correlation between TAP and the oncological outcome of HCC after radical hepatectomy.

The objective of this study was to explore the potential relationship between TAP and the prognosis of $\mathrm{HCC}$ after radical hepatectomy, and attempted to construct a robustly predictive nomogram on the strength of TAP and other prognostic variables of HCC patients.

\section{Methods}

\section{Patients}

The present study included 168 patients who had undergone curative surgery performed by the same surgeon during January 2018 to June 2020 at the Department of Hepatopancreatobiliary Surgery of Liaoning Cancer Hospital \& Institute. The recurrence group included 78 relapsed HCC patients, and the nonrecurrence group incorporated the other 90 patients. The inclusion criteria included: (1) aged 18-70 years; (2) had a single HCC; (3) received radical hepatectomy that was performed by the same surgical team and no other treatments, such as ablation therapy and immunotherapy; (4) had full clinicopathologic, TAP measure and follow-up information. Patients who had undergone postoperatively adjuvant treatments that were aimed at preventing the tumor recurrence were excluded. We also excluded patients who had other kinds of cancer history. The Liaoning Cancer Hospital \& Institute medical ethics committee had authorized this study.

\section{Data Collection And Tap Detection}

Based on our institute's medical records, we retrospectively collected the following clinical data: demographic characteristics (such as age, body mass index [BMI] and sex), biochemical variable (such as alanine aminotransferase [ALT], aspartate aminotransferase [AST], total bilirubin [TBIL], hepatitis B surface antigen [HBsAg]) and operative parameters (such as operative procedure, pringle maneuver, operation time and estimated blood loss). We collected information regarding maximum tumor diameter, 
tumor differentiation (poor or well), MVI (presence or not) and cirrhosis (yes or no) from the histopathological reports.

When TAP presents in blood, it will react with detection reagent (Abnormal Sugar Chain Structure of Glycoproteins) and generates a crystal-like condensation product. According to previous studies[13], TAPpositive was defined as condensation particle area $\geq 225 \mu \mathrm{m}^{2}$, TAP weakly positive was defined as the condensation particle area was between 121 and $225 \mu \mathrm{m}^{2}$, and as for TAP-negative, there was no crystallike condensation product. In the present study, high expression group has a condensation particle area that was $\geq 225 \mu \mathrm{m}^{2}$, and low expression group has a condensation particle area that was $<225 \mu \mathrm{m}^{2}$.

\section{Follow-up And Outcome}

According to clinical guidelines[14], all HCC patients who had undergone radical hepatectomy should receive regular outpatient visits. In general, all patients were recommended to visit outpatient every 3 months during the first year after hepatectomy, and at least every 6 months later. All patients received biochemical tests (such as serum alpha-fetoprotein, ALT, AST) and imaging examinations (such as contrast CT or MRI examination) during each follow-up. We obtained the oncology outcome of those who never receive outpatient visit at our hospital by telephone. The deadline was April 2021. The nonrecurrence group and recurrence group had the same median follow-up time, 21.5 months (interquartile range [IQR], 15.0-26.3) and 21.5 months (IQR, 15.0-28.0), respectively.

The primary aim of this study was to investigate whether there was a corroretion between TAP and the prognosis of HCC after radical hepatectomy. In addition, secondary purposes included identifying independent prognostic factors of RFS, defined as the interval time between radical resection and time to relapse, death or cut-off time (April 2021), and constructing a robustly predictive nomogram for postoperative HCC patients on basis of TAP and other prognostic variables.

\section{Statistical analysis}

Continuous variables were described as median (IQR), and difference between groups were tested by Mann-whitney U test. Dichotomous variables were summarized by frequency (percentage), and difference between groups was tested by Fisher's exact test or Chi-square test. Pearson r analysis was used to determine whether there was a correlation between maximum tumor size and TAP. Kaplan-Meier method was applied to calculate RFS, and the difference between groups was tested by log-rank test. The prognostic factors of RFS were determined by univariate and multivariate Cox regression model. All statistical analyses were performed by SPSS 22.0 software, and 2-sided, $p<0.05$ was considered statistically significant. We used EmpowerStats software to construct a nomogram, and an internal validation was applied to estimate its performance. A calibration plot was used to calculate calibration of this nomogram. The clinical value of this predictive model was evaluated by decision curve analysis.

We followed the methods of Huayong Cai et al. 2020[15]. 


\section{Results}

\section{Patient characteristics}

We retrospectively incorporated 168 patients with a single $\mathrm{HCC}$, who had undergone radical hepatectomy by the same surgeon during January 2018 to June 2020. On the basis of oncology status during followup, study population was divided into nonrecurrence group and recurrence group, incorporating 90 and 78 patients, respectively. Recurrence group had significantly higher TAP $(204.5[154.1,228.1]$ vs $165.1[134.9 \varangle 203.0] \mu \mathrm{m}^{2}$, respectively; $\left.p=0.002\right)$ and distinctly bigger tumor size $(5.0[3.5,6.0]$ vs $4.5[3.0$, $5.0] \mathrm{cm}$, respectively; $p=0.003$ ) than patients in nonrecurrence group (Table 1). However, in terms of demographic characteristics, HBsAg status, MVI, cirrhosis, liver function level, degree of differentiation, operative parameters and Child-Pugh score, no statistical difference was found between groups $(P>0.05$ for all) (Table 1).

The outcome of Pearson $r$ analysis between TAP and maximum tumor size was showed in Fig. 1. There was no significant relationship between TAP and maximum tumor size $(r=0.122 ; \mathrm{P}=.117$; Fig. 1). 
Table 1

Baseline characteristics in recurrence group and non-recurrence group

\begin{tabular}{|c|c|c|c|}
\hline & Recurrence group & Non-recurrence group & $P$ \\
\hline Characteristics & $(n=78)$ & $(n=90)$ & \\
\hline Age, year & $53.0(45.8,59.0)$ & $53.0(48.0,63.0)$ & 0.302 \\
\hline Gender(Male/Female) & $59 / 19$ & $72 / 18$ & 0.497 \\
\hline $\mathrm{HBsAg}(+/-)$ & $56 / 22$ & $65 / 25$ & 0.951 \\
\hline Cirrhosis(Yes/No) & $56 / 22$ & $67 / 23$ & 0.699 \\
\hline Differentiation(Poor/Well) & $35 / 43$ & $25 / 63$ & 0.092 \\
\hline Child-Pugh(A/B) & $76 / 2$ & $86 / 4$ & 0.687 \\
\hline AST (U/L) & $33 \cdot 3(24.0,88.0)$ & $27.5(22.4,45.0)$ & 0.078 \\
\hline $\mathrm{ALT}(\mathrm{U} / \mathrm{L})$ & $38.1(17.9,74.9)$ & $29.1(18.9,53.3)$ & 0.254 \\
\hline TBIL (U/L) & $14.5(10.6,22.9)$ & $14.2(11.0,19.5)$ & 0.515 \\
\hline $\mathrm{BMI}\left(\mathrm{kg} / \mathrm{m}^{2}\right)$ & $22.7(20.8,26.4)$ & $24.1(21.8,26.7)$ & 0.28 \\
\hline Tumor diameter (cm) & $5.0(3.5,6.0)$ & $4.5(3.0,5.0)$ & 0.003 \\
\hline MVI (Yes/No) & $36 / 42$ & $30 / 60$ & 0.09 \\
\hline $\operatorname{TAP}\left(\mu \mathrm{m}^{2}\right)$ & $204.5(154.1,228.1)$ & $165.1(134.9,203.0)$ & 0.002 \\
\hline Pringle maneuver (Yes/No) & $42 / 36$ & $50 / 40$ & 0.824 \\
\hline Total blood loss (ml) & $400.0(200.0,700.0)$ & $400.0(200.0,600.0)$ & 0.567 \\
\hline Operation time (min) & $220.0(200.0,185.0)$ & 247.5(203.8,300.0) & 0.214 \\
\hline Operative procedure & & & 0.902 \\
\hline Extended hemihepatotectomy & 7 & 12 & \\
\hline Hemihepatotectomy & 15 & 17 & \\
\hline Sectionectomy & 30 & 35 & \\
\hline Segmentectomy & 14 & 13 & \\
\hline Laparoscopic approach & 12 & 13 & \\
\hline
\end{tabular}


According to the cut-off value of TAP level mentioned in previous literatures[13], the whole population was categorized into the TAP high group (TAP $\geq 225 \mu \mathrm{m}^{2}$ ) and TAP low group (TAP $<225 \mu \mathrm{m}^{2}$ ). As shown in Fig. 2A, the RFS rate was distinctly higher in the TAP low group than TAP high group in the whole population $(P<0.001)$. Furthermore, in the stratification analysis according to maximum tumor diameter ( $\leq 5$ or $>5 \mathrm{~cm}$ ), the RFS rate of TAP high group was distinctly different from that of TAP low group $(P<0.001$ and $P=0.001$, respectively; Fig. $2 B, C)$.

In univariate analysis, TAP $(P<0.001)$, presence of MVI $(P=0.008)$, large tumor size $(P<0.001)$ and degree of tumor differentiation ( $p=0.042)$ were distinctly associated with RFS in HCC patients who had undergone radical surgery (Table 2). In addition, the multivariate analysis suggested that TAP (hazard ratio $[\mathrm{HR}$ ], 3.47; $95 \% \mathrm{Cl}, 2.18-5.51 ; \mathrm{P}<0.001)$, large tumor size (HR, 2.18; 95\% Cl, 1.36-3.49; $\mathrm{P}<0.001)$, poor tumor differentiation $(\mathrm{HR}, 0.53 ; 95 \% \mathrm{Cl}, 0.33-0.84 ; \mathrm{P}=0.007)$ and presence of $\mathrm{MVI}(\mathrm{HR}, 2.03 ; 95 \% \mathrm{Cl}, 1.28-$ $3.22 ; P=0.003$ ) were still independently correlated with RFS (Table 2 ). 
Table 2

Independent prognostic factors predicting RFS in the whole population

Characteristics Univariate analysis

$\mathrm{HR}(95 \% \mathrm{Cl})$

$0.70(0.42-1.19)$

0.189

Age, year

$\leq 60, \varangle 60$

Gender

Male/Female

HBsAg

Positive/Negative

$0.85(0.52-1.40)$

0.528

Cirrhosis

Yes/No

$0.99(0.61-1.63)$

0.979

Differentiation

Poor/Well

$0.63(0.40-0.98)$

0.042

$0.53(0.33-0.84)$

0.007

Child-Pugh

A/B

$0.82(0.20-3.32)$

0.776

MVI

Yes/No

$1.83(1.17-2.87)$

0.008

$2.03(1.28-3.22)$

0.003

Pringle maneuver

Yes/No

$1.13(0.73-1.77)$

0.583

Maximum tumor size $(\mathrm{cm})$

$\leq 5, \varangle 5$

$2.24(1.43-3.49)$

$\llbracket 0.001$

$2.18(1.36-3.49)$

$\varangle 0.001$

AST (U/L)

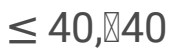

$1.22(0.77-1.94)$

0.389

$\operatorname{ALT}(\mathrm{U} / \mathrm{L})$

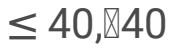

$1.36(0.87-2.12)$

0.178

$\operatorname{TBIL}(\mathrm{U} / \mathrm{L})$

$\leq 21, \varangle 21$

$1.19(0.72-1.99)$

0.494

$H B s A g$ hepatitis B surface antigen, $A L T$ alanine aminotransferase, $A S T$ aspartate aminotransferase, $T B I L$ total bilirubin level, $M V I$ microvascular invasion, TAP tomor abnormal protein 
$\operatorname{TAP}\left(\mu \mathrm{m}^{2}\right)$

$\otimes 225, \geq 225$

$3.34(2.13-5.23)$

$\llbracket 0.001$

$3.47(2.18-5.51)$

$\varangle 0.001$

$H B s A g$ hepatitis B surface antigen, $A L T$ alanine aminotransferase, $A S T$ aspartate aminotransferase, $T B I L$ total bilirubin level, $M V I$ microvascular invasion, TAP tomor abnormal protein

\section{Construction Of A Robust Nomogram}

According to abovementioned independent prognostic variables, we constructed a robust nomogram (Fig. 3A). Figure 3B depicted the calibration curves of this nomogram, which indicated favorable consistency between the predicted and observed relapse risk in the whole population, and the result of the Hosmer-Lemeshow test suggested an excellent fit $(P=0.519)$. Table 3 listed the statistical parameters of this predictive model, such as accuracy, specificity and sensitivity.

To emphasize the contribution of TAP in nomogram, we used ROC curve analysis to compare the prognostic performance of model incorporating TAP, MVI, maximun tumor diameter and degree of tumor differentiation with the model that integrated maximun tumor diameter, degree of tumor differentiation and MVI only. With regard to RFS, the area under the ROC curve (AUC) of the model without TAP and the combined model was 0.68 and $0.77(p=0.022$; Fig. $3 \mathrm{C}$ and Table 3$)$, respectively, which indicated that the predictive performance of conjunctive model was more excellent than the model that didn't incorporate TAP.

Table 3

The predictive performance of prognostic models

\begin{tabular}{|lll|}
\hline Variable & Combined model & Model without TAP \\
\hline AUC $(95 \% \mathrm{Cl})$ & $0.77(0.69,0.84)$ & $0.68(0.60,0.77)$ \\
\hline Sensitivity, \% & 64.1 & 47.4 \\
\hline Specificity, \% & 80 & 83.3 \\
\hline Accuracy, \% & 72.6 & 66.7 \\
\hline Positive predictive value, \% & 73.5 & 71.2 \\
\hline Negative predictive value, \% & 72 & 64.7 \\
\hline Positive likelihood ratio & 3.21 & 2.85 \\
\hline Negative likelihood ratio & 0.45 & 0.63 \\
\hline Diagnostic odd ratio & 7.14 & 4.51 \\
\hline AUC area under the curve, Cl confidence interval & \\
\hline
\end{tabular}


The clinical value of this nomogram was evaluated by decision curve analysis (Fig. 3D). When the highrisk threshold was greater than $25 \%$ in the clinical decision, compared with none patient relapse pattern or all patients relapse pattern, both models is more benefit in predicting RFS. Furthermore, as for predicting RFS, the nomogram incorporating TAP, MVI, degree of tumor differentiation and maximun tumor diameter was more benefit than the model that only incorporated MVI, degree of tumor differentiation and maximun tumor diameter in this range.

\section{Discussion}

As far as we know, this study demonstrated the correlation between TAP and the prognosis of HCC patients who had undergone radical hepatectomy for the first time, and poor prognosis was correlated with higher TAP. In addition, the multivariate analysis revealed that MVI, tumor differentiation and maximum tumor size were independently associated with RFS except for TAP. According to these prognostic variables, we constructed a robust nomogram for RFS of the HCC patients, which had an excellently predictive performance.

TAP is a collective term for glycoproteins generated by various malignant tumors, and numerous previous literatures had revealed the correlation between TAP and the development of different kinds of cancers. Among these studies, the majority concentrated on the clinical utility of TAP in the early diagnosis of malignancies, such as digestive tract precancerous lesion, bladder cancer, endometrial cancer, colorectal cancer, papillary thyroid cancer and breast cancer[9, 10, 16-19]. In recent years, several studies suggested that there was a close relationship between TAP and the prognosis of patients with various cancers. For example, Wu et al [12]indicated that TAP could be regarded as a prognostic factor for NSCLC patients, and Yao et al[13] revealed that high preoperative TAP level could predict the poor outcome of patients with esophageal squamous cell carcinoma. In line with these findings, this study suggested that there was a positive correlation between higher preoperative TAP and prognosis of HCC after curative resection, and multivariate analysis confirmed that it was a significant prognostic factor of RFS.

As everyone knows, $\mathrm{MVI}$ and tumor size are distinctly associated with recurrence risk of HCC after radical hepatectomy[20-22].In addition, Yang et al indicated that there was a negative correlation between tumor differentiation and the survival of patients with solitary HBV-associated HCC after curative surgery[23]. In consistent with previous studies, our results demonstrated that tumor differentiation, MVI and tumor size were independent predictors of RFS in HCC patients who had received radical treatment. As mentioned above, the median maximun tumor size was smaller in the nonrecurrence group than that in the recurrence group, but the potential correlation between maximum tumor diameter and TAP was excluded by Kaplan-Meier analysis and Pearson $r$ analysis. In addition, the predictive capability of the nomogram model that integrated TAP, tumor differentiation, MVI and maximun tumor size was more excellent than the model incorporating tumor differentiation, MVI and maximun tumor size only. In a word, there was no relationship between maximum tumor size and TAP, and TAP made a significant contribution in the nomogram model of RFS. 
It is worth noting that the present study had several limitations. First, this study retrospectively included patients from a single center, so we must notice some inevitable shortcomings. Second, our study population was relatively small, which might reduce the credibility of our findings, and we only focused on short-term survival. Therefore, a prospective, multicenter study is warranted to verify the results of our study.

\section{Conclusions}

In summary, as far as we know, the present study suggested that higher preoperative TAP was correlated with undesirable prognosis in HCC patients who had undergone radical hepatectomy, and on the strength of prognostic variables identified by multivariate analysis, we constructed a robust nomogram for RFS of postoperative HCC patients.

\section{Abbreviations}

TAP

Tumor abnormal protein

RFS

Recurrence-free survival

MVI

Microvascular invasion

$\mathrm{HCC}$

Hepatocellular cancer

BMI

Body mass index

ALT

Alanine aminotransferase

AST

Aspartate aminotransferase

TBIL

Total bilirubin

HBsAg

Hepatitis B surface antigen

\section{Declarations}

\section{Ethical Approval and consent to participate}

All procedures performed in studies involving human participants were in accordance with the ethical standards of the institutional and/or national research committee and with the 1964 Helsinki declaration and its later amendments or comparable ethical standards. 


\section{Consent for publication}

Not applicable

\section{Availability of data and materials}

The data used to support the findings of this study are available from the corresponding author upon request.

\section{Competing interests}

The authors declare that they have no conflict of interest.

\section{Funding}

Not applicable

\section{Authors' contributions}

Huayong Cai, Wenxin Li and Xiangdong Hua contributed to the conception of the study;

Huayong Cai, Wenxin Li and Yu Zhang performed the experiment;

Huayong Cai and Wenxin Li contributed significantly to analysis and manuscript preparation

Huayong Cai and Yu Zhang performed the data analyses and wrote the manuscript;

Xiangdong Huahelped perform the analysis with constructive discussions;

All authors read and approved the final manuscript.

\section{Acknowledgements}

Not applicable

\section{References}

1. Bray F, Ferlay J, Soerjomataram I, Siegel RL, Torre LA, Jemal A. Global cancer statistics 2018 : GLOBOCAN estimates of incidence and mortality worldwide for 36 cancers in 185 countries. CA Cancer J Clin. 2018;68:394-424.

2. European Association For The Study Of The. European Organisation For L, Treatment Of R. C: EASLEORTC clinical practice guidelines: management of hepatocellular carcinoma. J Hepatol 2012, 56:908-43.

3. Bhangui P, Allard MA, Vibert E, Cherqui D, Pelletier G, Cunha AS, Guettier C, Vallee JC, Saliba F, Bismuth H, et al: Salvage Versus Primary Liver Transplantation for Early Hepatocellular Carcinoma: 


\section{Do Both Strategies Yield Similar Outcomes? Ann Surg 2016, 264:155-163.}

4. Moniaux N, Andrianifahanana M, Brand RE, Batra SK. Multiple roles of mucins in pancreatic cancer, a lethal and challenging malignancy. Br J Cancer. 2004;91:1633-8.

5. Hakomori S. Glycosylation defining cancer malignancy: new wine in an old bottle. Proc Natl Acad Sci U S A. 2002;99:10231-3.

6. Hakomori S. Tumor malignancy defined by aberrant glycosylation and sphingo(glyco)lipid metabolism. Cancer Res. 1996;56:5309-18.

7. Meezan E, Wu HC, Black PH, Robbins PW. Comparative studies on the carbohydrate-containing membrane components of normal and virus-transformed mouse fibroblasts. II. Separation of glycoproteins and glycopeptides by sephadex chromatography. Biochemistry. 1969;8:2518-24.

8. Dube DH, Bertozzi CR. Glycans in cancer and inflammation-potential for therapeutics and diagnostics. Nat Rev Drug Discov. 2005;4:477-88.

9. Wu XY, Huang XE. Clinical Application of Serum Tumor Abnormal Protein (TAP) in Colorectal Cancer Patients. Asian Pac J Cancer Prev. 2015;16:3425-8.

10. Ma A, Fan D, Yan F. A study of the application of TAP combined with transvaginal ultrasound in the diagnosis of early-stage endometrial cancer. Oncol Lett. 2018;16:5186-90.

11. Lan F, Zhu M, Qi Q, Zhang Y, Liu Y. Prognostic value of serum tumor abnormal protein in gastric cancer patients. Mol Clin Oncol. 2016;5:216-20.

12. Cheng Y, Chen Y, Zang G, Chen B, Yao J, Zhang W, Wang H, Yu L, He P, Zhang Y, Wu H. Increased Expression of TAP Is Predictive of Poor Prognosis in Patients with Non-Small Cell Lung Cancer. Cancer Manag Res. 2020;12:1941-6.

13. Cheng Y, Fang Q, Chen Y, Zang G, Yao J. High Expression of Tumor Abnormal Protein Preoperatively Predicts Poor Prognosis of Patients With Esophageal Squamous Cell Carcinoma. Front Surg. 2021;8:609719.

14. Benson AB, D'Angelica MI, Abbott DE, Abrams TA, Alberts SR, Anaya DA, Anders R, Are C, Brown D, Chang DT, et al. Guidelines Insights: Hepatobiliary Cancers, Version 2.2019. J Natl Compr Canc Netw. 2019;17:302-10.

15. Cai H, Zhang Y, Zhang H, Cui C, Li C, Lu S. Prognostic role of tumor mutation burden in hepatocellular carcinoma after radical hepatectomy. J Surg Oncol. 2020;121:1007-14.

16. Sun C, Deng F, Meng L, Chen G. Correlation between TAP detection and common digestive tract precancerous lesions. Oncol Lett. 2018;15:1616-20.

17. Zhang L, Guo X, Min Y, Xu J. Tumor abnormal protein (TAP) examination contributes to primary diagnosis of bladder cancer. Int J Clin Exp Med. 2015;8:18528-32.

18. Liu Z, Cai J, Yu Y, Fang H, Si Y, Jankee JJ, Shen M. Tumor Abnormal Protein as a Novel Biomarker in Papillary Thyroid Carcinoma. Clin Lab. 2017;63:479-85.

19. Chen WX, Yang LG, Cheng L, Zhu YL. Tumor Abnormal Protein in the Diagnosis of Breast Cancer in Patients with a Palpable Mass. Ann Clin Lab Sci. 2019;49:297-301. 
20. Kokudo T, Hasegawa K, Matsuyama Y, Takayama T, Izumi N, Kadoya M, Kudo M, Ku Y, Sakamoto M, Nakashima 0 , et al. Survival benefit of liver resection for hepatocellular carcinoma associated with portal vein invasion. J Hepatol. 2016;65:938-43.

21. Cucchetti A, Djulbegovic B, Tsalatsanis A, Vitale A, Hozo I, Piscaglia F, Cescon M, Ercolani G, Tuci F, Cillo $U$, Pinna AD. When to perform hepatic resection for intermediate-stage hepatocellular carcinoma. Hepatology. 2015;61:905-14.

22. Bruix J, Cheng A-L, Meinhardt G, Nakajima K, De Sanctis Y, Llovet J. Prognostic factors and predictors of sorafenib benefit in patients with hepatocellular carcinoma: Analysis of two phase III studies. J Hepatol. 2017;67:999-1008.

23. Shen J, Liu J, Li C, Wen T, Yan L, Yang J. The Impact of Tumor Differentiation on the Prognosis of HBV-Associated Solitary Hepatocellular Carcinoma Following Hepatectomy: A Propensity Score Matching Analysis. Dig Dis Sci. 2018;63:1962-9.

\section{Figures}

\section{$r=0.149 p=0.053$}

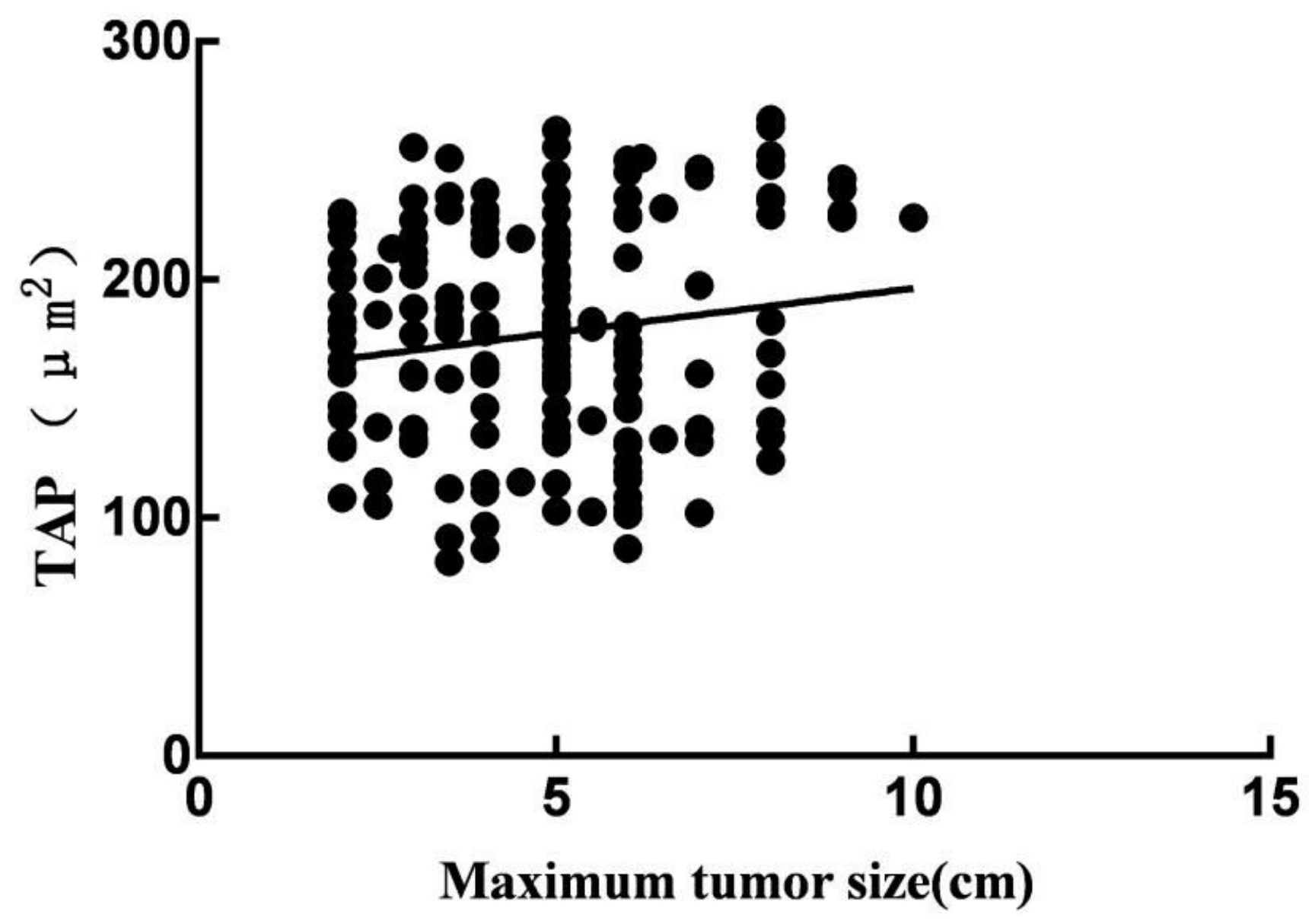


Figure 1

The correlations between TAP and maximum tumor size. There was no correlation between TAP and maximum tumor size. TAP tumor abnormal protein
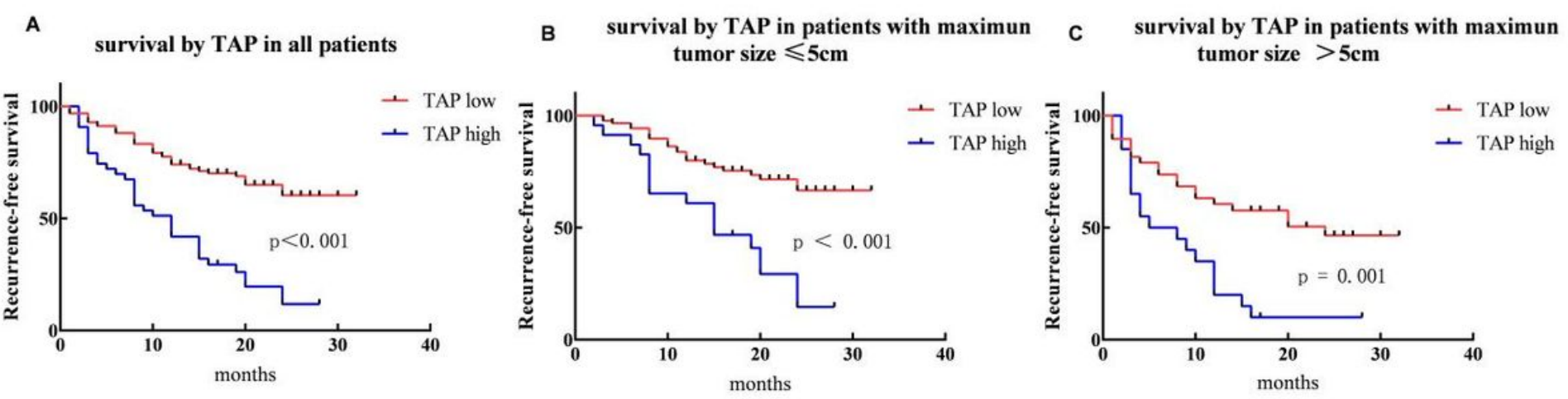

Figure 2

Kaplan-Meier curves of RFS. (A) Kaplan-Meier curves of RFS in the whole population by TAP. (B-C) Kaplan-Meier curves of RFS in patients with maximum tumor size $\leq 5 \mathrm{~cm}$ or $>5 \mathrm{~cm}$ by TAP. TAP tumor abnormal protein, RFS recurrence-free survival 
A

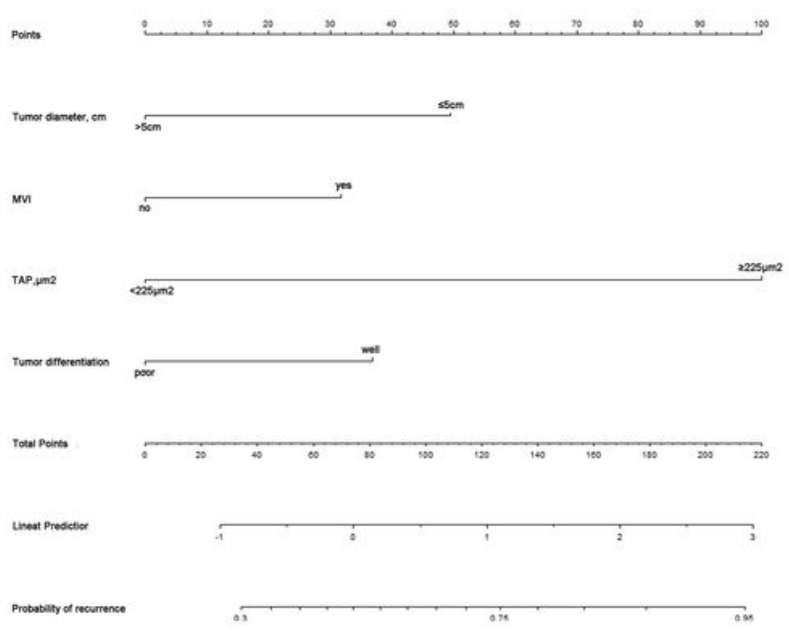

c

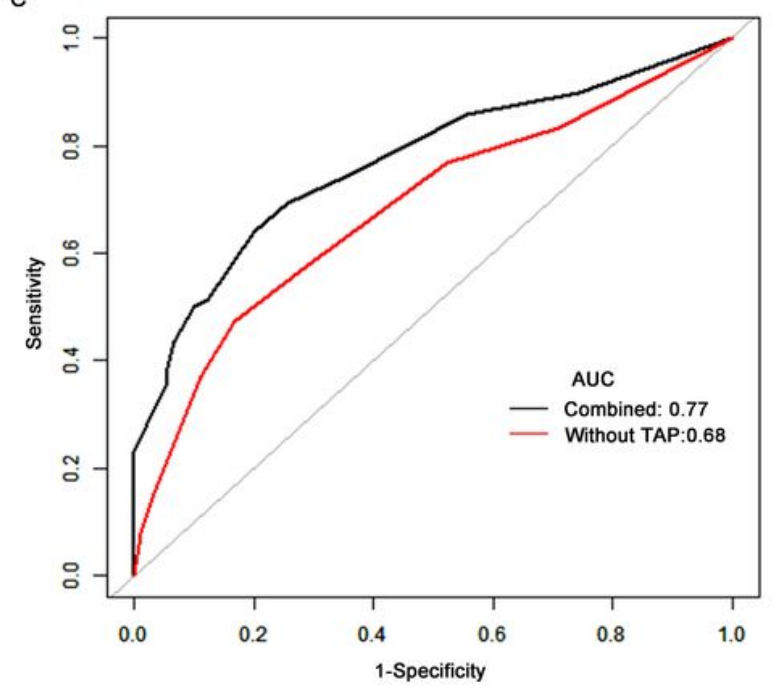

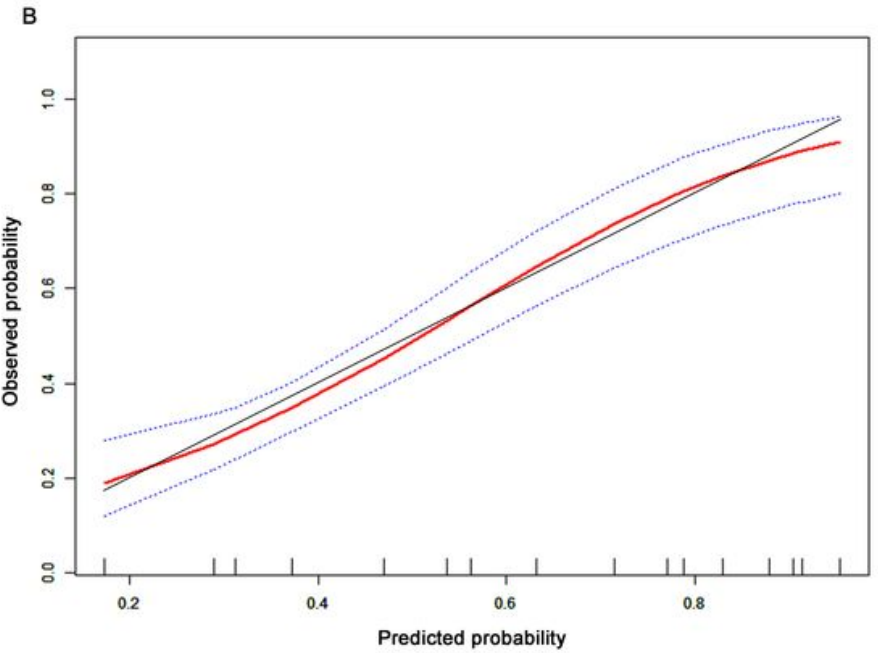

D

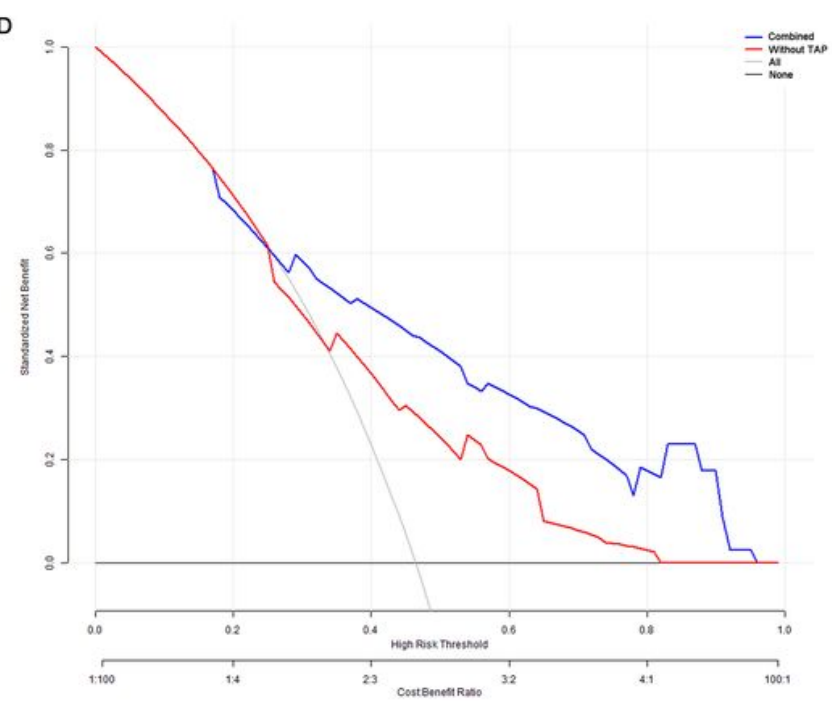

\section{Figure 3}

(A) A reliable nomogram for recurrence-free survival in HCC patients who had received radical hepatectomy. (B) Calibration curves of the nomogram in the whole cohort. The $45^{\circ}$ black line indicates optimal prediction. The red line means the predictive performance of the nomogram. The region between two blue dotted line represents $95 \%$ confidence interval of the nomogram. (C) Receiver operating characteristic curves of the combined model (integrate TAP, tumor differentiation, tumor diameter and $\mathrm{MVI}$ ) and model without TAP. (D) Decision curve analysis for recurrence-free survival. Black line: none patient recurrence. Gray line: all patients recurrence. Red line: model without TAP. Blue line: combined model. TAP tumor abnormal protein, MVI microvascular invasion 